\title{
INTERAÇÃO ENTRE MICORRIZAS ARBUSCULARES E ÁCIDO GIBERÉLICO NO DESENVOLVIMENTO VEGETATIVO DE PLANTAS DE CITRANGE CARRIZO ${ }^{1}$
}

\author{
EFFECT OF ARBUSCULAR MYCORRHIZAE AND GIBBERELIC ACID INTERACTION \\ ON VEGETATIVE GROWTH OF CARRIZO CITRANGE SEEDLINGS
}

\author{
Paulo Vitor Dutra de Souza ${ }^{2}$
}

\section{RESUMO}

As plantas cítricas são, geralmente, dependentes dos fungos micorrízicos arbusculares (FMA). Há relatos indicando a existência de um intercâmbio hormonal entre as plantas e os FMA e também a comprovação de que a simbiose planta-FMA é beneficiada pela aplicação radicular de auxinas. Com o objetivo de verificar se esta interação também ocorre com outros grupos de fitorreguladores, submergiu-se, por 10 segundos, o sistema radicular de plântulas de citrange Carrizo (Citrus sinensis (L.) Osb. X Poncirus trifoliata (L.) Raf.), previamente inoculadas, ou não, com Glomus intraradices Schenck \& Smith, em uma solução de 10ppm de ácido giberélico $\left(A G_{3}\right)$. Após sete meses de cultivo em casa de vegetação, verificou-se que os FMA incrementaram o desenvolvimento vegetativo do citrange Carrizo, além de provocarem um aumento nos níveis foliares de $\mathrm{P}, \mathrm{Zn}$ e $\mathrm{Cu}$ e induzirem a um decréscimo no conteúdo de $\mathrm{Ca}, \mathrm{Mg}$, Fe e Mn nos tecidos. Os FMA não afetaram os níveis foliares de $\mathrm{N}$ e $\mathrm{K}$. $\mathrm{O} \mathrm{AG}_{3}$ somente aumentou o diâmetro do colo das plantas, não modificando sua altura, peso seco da parte aérea e raízes, número de folhas e superfície foliar. $\mathrm{O} \mathrm{AG}_{3}$ incrementou os níveis de $\mathrm{P}$ e Fe nos tecidos foliares, reduziu os teores de Ke não modificou os teores dos demais nutrientes. $O \mathrm{AG}_{3}$ não alterou a colonização radicular com FMA. No entanto, verificou-se uma interação positiva e significativa entre os FMA e o $A G_{3}$ sobre o desenvolvimento vegetativo das plantas de citrange Carrizo, indicando que o $A_{3}$ favorece a simbiose, permitindo uma redução no período de produção da muda.

Palavras-chave: endomicorrizas, citros, reguladores de crescimento.

\section{SUMMARY}

Citrus plants generaly depend on arbuscular mycorrhizal fungi $(A M F)$. There are several reports indicating interaction between hormones and AMF. It has also been proven

\begin{abstract}
that plant and AMF symbiosis benefits from auxin aplication to roots. The present study had the objective to determine if planthormone interaction occurred with hormone groups other than auxins. Mycorrhizal and nonmycorrhizal Carrizo citrange (Citrus sinensis (L.) Osb. X Poncirus trifoliata (L.) Raf.) root systems were submerged for 10 seconds in a $10 \mathrm{ppm}$ gibberelic acid $\left(\mathrm{GA}_{3}\right)$ solution. AMF increased the vegetative growth of Carrizo citrange seedlings as well as $P, \mathrm{Zn}$ and $\mathrm{Cu}$ leaf contents. Mycorrhizal seedlings had lower $\mathrm{Ca}, \mathrm{Mg}, \mathrm{Fe}$ and $\mathrm{Mn}$ concentrations in leaves than nonmycorhizal seedlings, while $N$ and $K$ concentrations were not affected by $A M F . G_{3}$ treated seedlings had a bigger trunk diameter but the treatment did not increase seedling height, dry weight, leaf number and leaf area per plant. $G A_{3}$ treatment also resulted in increased levels of $P$ and $F e$ in the leaves, reduced $K$ leaf contents but had no effect on other nutrients and did not affect root colonization by $A M F$. Moreover, the interaction between $A M F$ and $G A_{3}$ had a significative effect on Carrizo citrange vegetative growth, indicating that $G A_{3}$ favored plant-AMF symbiosis.
\end{abstract}

Key words: endomycorrhizal fungi, citrus, growth regulators.

\section{INTRODUÇÃO}

Fungos micorrízicos arbusculares (FMA) são organismos que se associam simbioticamente às raízes das plantas, incrementando a absorção nutricional e estimulando o crescimento destas (TOBAR et $\boldsymbol{a l}$. , 1994). As plantas cítricas são, normalmente, altamente dependentes dos FMA (DUTRA et al., 1995). Portanto, a aplicação desses fungos tem grande potencial naquelas áreas onde os mesmos estão

\footnotetext{
${ }^{1}$ Extraído da Tese de Doutorado apresentada à Universidad Politécnica de Valencia (Espanha).

${ }^{2}$ Engenheiro Agrônomo, Doutor, Departamento de Horticultura e Silvicultura, Faculdade de Agronomia, Universidade Federal do Rio Grande do Sul, Caixa Postal 776, 91501-970, Porto Alegre-RS. 
ausentes (PALAZZO et al., 1992) ou quando o cultivo é realizado em substratos estéreis. Este é o caso da multiplicação de plantas em recipientes.

Fungos ectomicorrízicos têm a capacidade de produzir auxinas, giberelinas e citocininas (CRAFT \& MILLER, 1974; BARROSO et al., 1986; HANLEY \& GREENE, 1987), mas a síntese de tais fitorreguladores por endomicorrizas tem sido pouco estudada, devido à dificuldade de multiplicar esse fungo em meios de cultura artificiais (COOPER, 1984). Há evidências de que os fitorreguladores estão correlacionados com a interação planta-FMA, pois encontrou-se incremento na atividade citocinínica em plantas de laranjeira azeda (Citrus aurantium L.), quando estas foram inoculadas com FMA (EDRISS et al., 1984). Além disso, demonstrou-se que a aplicação de AIB nas raízes incrementou o desenvolvimento vegetativo de plantas inoculadas com FMA, não sendo verificado o mesmo comportamento em ausência do endófito (DUTRA et al., 1996).

A resposta das plantas à aplicação exógena de fitorreguladores é variável com seu estado fisiológico. A maioria das giberelinas, quando aplicadas durante o crescimento ativo, são metabolizadas com rapidez, originando produtos inativos. Além do mais, podem facilmente ser convertidas em conjugados normalmente inativos (SALISBURY \& ROSS, 1994). Portanto, a aplicação de ácido giberélico na fase de elongação radicular, supostamente uma fase em que a planta não responde à aplicação exógena desse fitorregulador, pode trazer novas informações a respeito da interação fitorreguladoresFMA. A eficiência dos FMA é variável com a espécie em estudo. Nesse sentido, Glomus intraradices mostrou-se muito eficiente em simbiose com plantas cítricas na região mediterrânea espanhola, onde foi realizado este estudo (CAMPRUBÍ, 1994).

Neste trabalho, estuda-se a interação entre a aplicação exógena de ácido giberélico em uma fase de crescimento ativo e a inoculação com FMA (Glomus intraradices) em plantas de citrange Carrizo, visando à redução do período necessário à produção da muda.

\section{MATERIAL E MÉTODOS}

Este estudo foi realizado em casa de vegetação de polietileno, situada na localidade de Alcanar (Tarragona, Espanha). No mês de março de 1994, semeou-se citrange Carrizo (Citrus sinensis (L.) Osb. X Poncirus trifoliata (L.) Raf.) em bandejas de plástico de 20 litros. O substrato utilizado foi uma mistura de areia silícea, perlita e turfa Sphagnum (3:2:1, em volume), com pH 6,8 e fertilizado com Osmocote Plus ${ }^{\circledR}$ (fertilizante de liberação lenta; à base de N,P,K; de 2 a 3 meses de duração; 6 gramas/litro de substrato).

No momento da semeadura, adicionaramse, à metade das bandejas, 6 gramas/semente de raízes e solo rizosférico de alfafa (Medicago sativa L.) cv. Aragón, colonizados com o fungo micorrízico Glomus intraradices Schenck \& Smith. À outra metade das bandejas, adicionaram-se 6 gramas/semente de uma mistura de raízes e solo rizosférico da mesma cultivar de alfafa produzida em ausência de propágulos de FMA.

No mês de junho do mesmo ano, efetuouse o transplante dos porta-enxertos para sacos de polietileno preto perfurados (5 litros). O substrato empregado nesse momento foi uma mistura de turfa preta e turfa Sphagnum (1:1, em volume), adquirido já misturado sob o nome comercial de "T Plant Sustrat".

O delineamento experimental foi em blocos ao acaso com quatro tratamentos, resultantes da combinação de duas doses (0 e 10ppm) de ácido giberélico $\left(\mathrm{AG}_{3}\right)$ e a inoculação ou não com micorrizas arbusculares, três repetições e vinte plantas por parcela. $\mathrm{O} \mathrm{AG}_{3}$ foi aplicado mediante imersão do sistema radicular por um período de 10 segundos, em uma solução de 10ppm. As raízes das plantas testemunhas foram imersas pelo mesmo período em água destilada.

Em outubro, avaliou-se o desenvolvimento vegetativo das plantas. Mediram-se a altura das plantas (desde o colo até o ápice) e o diâmetro do colo, o número de folhas por planta e a área foliar por folha. A área foliar foi medida pelo uso do aparelho "LI-Cor LI-3000 Area Meter". As plantas tiveram sua parte aérea e radicular separadas, lavadas com água destilada e postas a secar em estufa a $65^{\circ} \mathrm{C}$ até peso constante. Após, mediu-se o peso seco dessas frações. A parte aérea foi moída em moinho e enviada para análise do conteúdo nutricional foliar no Instituto Valenciano de Investigaciones Agrárias (IVIA; Montcada, Valencia, Espanha). O N total foi determinado pelo método semi-micro-Kjeldahl (BREMMER, 1965) utilizando um analisador semiautomático Tecator. O P foi determinado colorimetricamente (FISKE \& SUBBAROW, 1925) e o K, $\mathrm{Ca}, \mathrm{Mg}, \mathrm{Fe}, \mathrm{Mn}, \mathrm{Zn}$ e $\mathrm{Cu}$, por fotômetro de chamas Perkin-Elmer (CHAPMAN \& PRATT, 1973).

No mesmo período, determinou-se a intensidade de colonização micorrízica, coletando-se duas raízes secundárias por planta de cada planta na parcela. Estas foram lavadas com água destilada e cortadas em segmentos de $1 \mathrm{~cm}$ de comprimento. A seguir, separaram-se, ao acaso, 30 segmentos por repetição ( 90 por tratamento), que foram clarificados e tingidos segundo adaptações ao método descrito 
por PHILLIPS \& HAYMAN (1970). Após, estes foram montados em lâminas de vidro e examinados em microscópio para avaliar a presença e intensidade de hifas, vesículas e arbúsculos, segundo método descrito por NEMEC (1992). A porcentagem de raízes colonizadas foi obtida do número de segmentos infectados em relação ao total analisado. Para determinar a densidade de hifas, atribuiu-se o valor 0 para ausência de estruturas; 1, para presença fraca; 2, para presença moderada; e 3, para presença intensa. A densidade de vesículas e arbúsculos também foi relacionada com uma escala de 0 a 3 , onde se considerou como 0 a ausência de estruturas; 1, para 1 a 50 estruturas; 2, para 51 a 100; e 3, para mais de 100.

\section{RESULTADOS E DISCUSSÃO}

Na tabela 1, são apresentados os dados referentes à altura e diâmetro do colo das plantas. Esses parâmetros foram afetados significativamente pela aplicação de $\mathrm{AG}_{3}$ e pelos FMA. Verificou-se uma interação significativa entre esses fatores. A aplicação de $\mathrm{AG}_{3}$ não alterou a altura das plantas não micorrizadas, porém incrementou o diâmetro do colo das mesmas. As plantas não tratadas com $\mathrm{AG}_{3}$ apresentaram uma maior altura e diâmetro do colo quando inoculadas com FMA. A aplicação de $\mathrm{AG}_{3}$ incrementou o efeito dos FMA.

O número de folhas e a superfície foliar não foram alterados pela aplicação de $\mathrm{AG}_{3}$, mas sim pelos FMA (Tabela 2). O número de folhas passou de aproximadamente 23 por planta em ausência, para 40 folhas por planta em presença de FMA. A superfície das folhas das plantas não inoculadas foi
Tabela 2 - Efeito da aplicação radicular de ácido giberélico $\left(\mathrm{AG}_{3} ; 10 \mathrm{ppm}\right)$ e da inoculação com fungos micorrízicos arbusculares (FMA) sobre o número de folhas e a superfície por folha de plantas de citrange Carrizo.

\begin{tabular}{|c|c|c|c|c|c|c|}
\hline \multirow[b]{2}{*}{ Tratamento } & \multicolumn{3}{|c|}{ Número de folhas/planta $(\mathrm{g})$} & \multicolumn{3}{|c|}{ Superfície/folha $\left(\mathrm{cm}^{2}\right)$} \\
\hline & + FMA & - FMA & Média & + FMA & - FMA & Média \\
\hline$+\mathrm{AG}_{3}{ }^{1}$ & 41,36 & 29,90 & 35,63 & 8,85 & 5,21 & 7,03 \\
\hline$-\mathrm{AG}_{3}$ & 39,45 & 22,80 & 31,25 & 7,10 & 4,52 & 5,81 \\
\hline Média & ${ }^{2} \mathrm{~A} 40,40$ & B 26,35 & & A 7,97 & B 4,86 & \\
\hline
\end{tabular}

$\mathrm{AG}_{3}=$ ácido giberélico

Letras maiúsculas diferentes na mesma linha indicam diferença significativa pelo teste DMS em 5\% de probabilidade de erro.

de $4,9 \mathrm{~cm}^{3}$, enquanto que nas inoculadas com FMA foi de $7,0 \mathrm{~cm}^{2}$. Como conseqüência do ocorrido para altura, diâmetro do colo, número de folhas por planta e superfície foliar, o peso seco das raízes e da parte aérea também foram influenciados pelo $\mathrm{AG}_{3} \mathrm{e}$ pelos FMA (Tabela 1). A interação entre ambos os fatores foi significativa. $\mathrm{O} \mathrm{AG}_{3}$ foi ineficaz em plantas não micorrizadas, mas em presença de FMA, interagiu com estes, incrementando significativamente o peso seco de raízes e parte aérea das plantas. Os resultados aqui encontrados confirmam a dependência dos citros aos FMA, em concordância com outros experimentos (DUTRA et al., 1995; SOUZA et al., 1996; SOUZA et al., 1997).

As giberelinas têm a capacidade de estimular o crescimento generalizado em plantas. No entanto, quando aplicadas durante o crescimento ativo, a maioria delas são metabolizadas com rapidez, originando produtos inativos. Além do mais, podem facilmente ser convertidas em conjugados normalmente inativos (SALISBURY \& ROSS, 1994), o que poderia explicar os resultados aqui encontrados.

A aplicação de $\mathrm{AG}_{3}$ não alterou os conteúdos foliares de $\mathrm{N}$, $\mathrm{Mg}, \mathrm{Mn}, \mathrm{Zn}$ e $\mathrm{Cu}$; e incrementou os conteúdos de P e Fe (Tabela 3). Já, os FMA não alteraram os níveis foliares de N (Tabela 3) e K (Tabela 4), incrementaram os de $\mathrm{P}, \mathrm{Zn}$ e $\mathrm{Cu}$ (Tabela 3) e reduziram os de $\mathrm{Ca}$

${ }^{2}$ Letras maiúsculas diferentes na mesma linha e letras minúsculas diferentes na mesma coluna indicam diferença significativa pelo teste DMS em $5 \%$ de probabilidade de erro.

$* ; * *=$ significativo a $5 \%$ e $1 \%$, respectivamente. 
Tabela 3 - Efeito da aplicação radicular de ácido giberélico $\left(\mathrm{AG}_{3} ; 10 \mathrm{ppm}\right)$ e da inoculação com fungos micorrízicos arbusculares (FMA) sobre o conteúdo em macro e micronutrientes de folhas de citrange Carrizo.

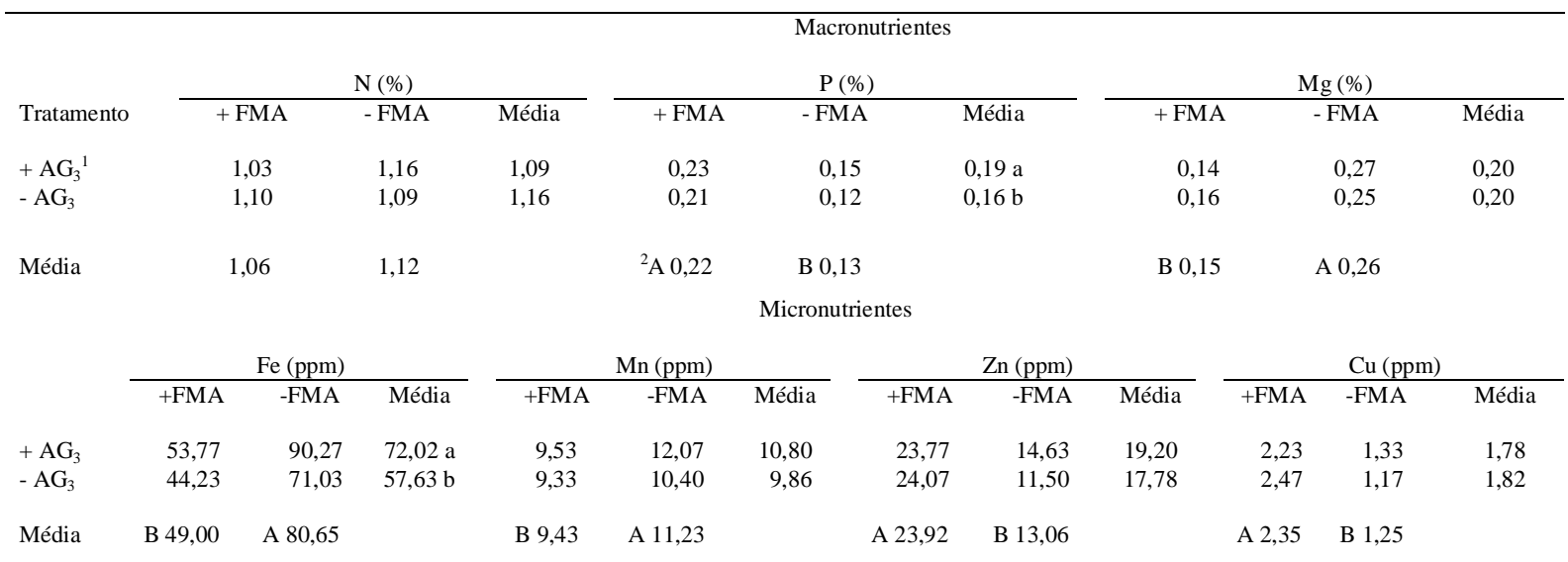

${ }^{1} \mathrm{AG}_{3}=$ ácido giberélico.

${ }^{2}$ Letras maiúsculas diferentes na mesma linha e letras minúsculas diferentes na mesma coluna indicam diferença significativa pelo teste DMS em $5 \%$ de probabilidade de erro.

(Tabela 4), Mg, Fe e Mn (Tabela 3). A interação $\mathrm{AG}_{3}$ x FMA somente foi significativa para os conteúdos de $\mathrm{K}$ e Ca. Verificou-se que a aplicação de $\mathrm{AG}_{3}$ reduziu os conteúdos de $\mathrm{K}$ foliar, sendo mais acentuado em ausência de FMA. As plantas micorrizadas apresentaram menores conteúdos foliares de $\mathrm{Ca}$. Nas plantas tratadas com $\mathrm{AG}_{3}$, o conteúdo foliar de $\mathrm{Ca}$ foi semelhante ao destas, independentemente dos FMA.

O efeito positivo dos FMA sobre o acúmulo de $\mathrm{P}$ pelas plantas confirma resultados anteriores e se deve à presença do micélio externo dos FMA, o qual tem importante papel na absorção de nutrientes de difusão lenta, como o fósforo (TOBAR et al., 1994). Neste trabalho, à semelhança de outros

Tabela 4 - Efeito da aplicação radicular de ácido giberélico $\left(\mathrm{AG}_{3}\right.$; $10 \mathrm{ppm})$ e da inoculação com fungos micorrízicos arbusculares (FMA) sobre o conteúdo foliar em potássio e cálcio de plantas de citrange Carrizo.

\begin{tabular}{|c|c|c|c|c|}
\hline \multirow[b]{2}{*}{ Tratamento } & \multicolumn{2}{|c|}{$\mathrm{K}(\%)$} & \multicolumn{2}{|c|}{$\mathrm{Ca}(\%)$} \\
\hline & + FMA & - FMA & + FMA & - FMA \\
\hline $\begin{array}{l}+\mathrm{AG}_{3}{ }^{1} \\
-\mathrm{AG}_{3}\end{array}$ & $\begin{array}{l}1,07 \mathrm{~b} \\
1,11 \mathrm{a}\end{array}$ & $\begin{array}{l}1,03 \mathrm{~b} \\
1,14 \mathrm{a}\end{array}$ & $\begin{array}{r}0,90 \\
{ }^{2} \mathrm{~B} 0,93\end{array}$ & $\begin{aligned} & 0,89 \mathrm{~b} \\
& \text { A } 1,14 a\end{aligned}$ \\
\hline $\begin{array}{l}\text { Efeito } \mathrm{AG}_{3} \\
\text { Efeito FMA } \\
\text { Interação }\end{array}$ & & & & $*$ \\
\hline
\end{tabular}

${ }^{1} \mathrm{AG}_{3}=$ ácido giberélico.

${ }^{2}$ Letras maiúsculas diferentes na mesma linha e letras minúsculas diferentes na mesma coluna indicam diferença significativa pelo teste DMS em 5\% de probabilidade de erro.

*; **;NS $=$ significativo a $5 \%, 1 \%$ e não significativo, respectivamente.
(ROSE \& YOUNGBERG, 1981; AL-WHAIBI \& KHALIEL, 1994), os FMA não alteraram os níveis de $\mathrm{N}$ e $\mathrm{K}$ foliares. Por outro lado, as plantas micorrizadas podem ver diminuída a absorção de certos elementos minerais. Esse é o caso de metais pesados (MOSSE, 1986) ou Mn (ARINES et al., 1989). Resposta semelhante pode ser encontrada com relação ao Ca e Mg, sendo atribuída a um efeito tampão dos FMA (AZCÓN et al.,1991; AZCÓN \& BAREA, 1992).

Apesar de ter havido uma interação significativa entre $\mathrm{AG}_{3}$ e FMA, a aplicação de $\mathrm{AG}_{3}$ não alterou a percentagem de colonização, nem o número de estruturas de FMA das raízes de citrange Carrizo (Tabela 5). Esse resultado evidencia que os FMA utilizam, direta ou indiretamente, o $\mathrm{AG}_{3}$, cul-

Tabela 5 - Efeito da aplicação radicular de ácido giberélico $\left(\mathrm{AG}_{3}\right.$; $10 \mathrm{ppm}$ ) sobre a intensidade de colonização radicular com micorrizas arbusculares em citrange Carrizo.

\begin{tabular}{lcccc}
\hline & \multirow{2}{*}{ Colonização (\%) } & \multicolumn{3}{c}{ Estruturas $^{2}$} \\
\cline { 3 - 5 } Tratamento & & Hifas & Vesículas & Arbúsculos \\
& & & & \\
\hline & & & & \\
$+\mathrm{AG}_{3}{ }^{1}$ & 73,87 & 1,45 & 0,19 & 1,11 \\
$-\mathrm{AG}_{3}$ & 80,88 & 1,51 & 0,25 & 0,88 \\
& & & & \\
Signif. & $\mathrm{NS}$ & $\mathrm{NS}$ & $\mathrm{NS}$ & $\mathrm{NS}$ \\
\hline
\end{tabular}

${ }^{1}$ Ácido giberélico

${ }^{2}$ Atribuíram-se índices de 0 a 3 para cada segmento de $1 \mathrm{~cm}$ de raiz secundária.

NS: não significativo em nível de $5 \%$ de probabilidade pelo teste DMS. 
minando com uma maior velocidade de crescimento das plantas, possibilitando reduzir o período de produção da muda. Assim, o mutualismo planta-FMA também é favorecido pela aplicação de $\mathrm{AG}_{3}$, além de sê-lo pela aplicação de auxinas (DUTRA $\boldsymbol{e t} \boldsymbol{a l}$., 1995; SOUZA et al., 1997).

\section{CONCLUSÕES}

Os fungos micorrízicos arbusculares incrementam o desenvolvimento vegetativo de plantas de citrange Carrizo.

O ácido giberélico é ineficaz em promover o crescimento de plantas não micorrizadas, mas em presença de fungos micorrízicos arbusculares interage com este, incrementando significativamente a velocidade de crescimento das plantas, permitindo reduzir o período de produção da muda.

\section{AGRADECIMENTO}

À empresa VIVEROS GURBÍ S.A.T. (AlcanarTarragona-Espanha) pelo financiamento deste estudo, mediante convênio de colaboração com a Universidad Politécnica de Valencia (Espanha) (convênio n ${ }^{\circ}$ 60930028/70).

\section{REFERÊNCIAS BIBLIOGRÁFICAS}

AL-WHAIBI, M.H., KHALIEL, A.S. The effect of Mg on Ca, K and $\mathrm{P}$ content of date palm seedlings under mycorrhizal and nonmycorrhizal condictions. Mycoscience, Tokio, v.35, p.213-217, 1994.

ARINES, J., VILARIÑO, A., SAINZ, M. Effect of different inocula of vesicular-arbuscular mycorrhizal fungi on manganese content and concentration in red clover (Trifolium pratense L.) plants. New Phytologist, Cambridge, v.112, p.215-219, 1989.

AZCÓN, R., BAREA, J.M. The effect of vesicular-arbuscular mycorrhizae in decreasing $\mathrm{Ca}$ acquisition by alfalfa plants in calcareous solis. Biology and Fertility of Soils, Berlin, v.13, p.155-159, 1992 .

AZCÓN, R., RUBIO, R., BAREA, J.M Selective interactions between different species of mycorrhizal fungi Rhizobium melioti strains and their effects on growth, $\mathrm{N}_{2}$ fixation $\left({ }^{15} \mathrm{~N}\right)$ and nutrition of Medicago sativa L. New Phytologist, Cambridge, v.117, p.399-404, 1991.

BARROSO, J., NEVES, H.C., PAIS, M.S. Production of indole-3-ethanol and indole-3-acetic acid by the mycorrhizal fungus of Ophrys lutea (Orchidaceae). New Phytologist, Cambridge, v.103, p.745-749, 1986.

BREMMER, J. M. Total nitrogen. In: BLACK, C. A. (ed.) Methods of soil analisis. Madison : American Society of Agronomy, 1965. p.1149-1178.

CAMPRUBÍ, A. Micorrizas en viveros de cítricos: caracterización, selección de hongos y aplicación de esta biotecnologia en un sistema de producción en campo. Barcelona, 1994. 267p. Tese (Doutorado em Biologia). Faculdad de Biologia, Universidad de Barcelona, Barcelona, 1994.

CHAPMAN, M.D., PRATT, P.F. Métodos y análisis para suelos, plantas y águas. México : Trillas, 1973. 159p.
COOPER, K.M. Physiology of VA mycorrhizal associations. In: POWEL, C.L.; BAGYARAJ, J. (Eds.). VA Mycorrhiza. Boca Raton : CRC, 1984. p.155-186.

CRAFT, C.B., MILLER, C.O. Detection and quantification of citokinins produced by mycorrhizal fungi. Plant Physiology, Bethesda, v.54, p.586-588, 1974.

DUTRA, P.V., ALMELA, V., PONS, J., et al. Inoculación de patrones de cítricos con micorrizas vesiculares-arbusculares y su comportamiento en los suelos de vivero. Phytoma, Valencia, n.65, p.17-22, 1995.

DUTRA, P.V., ABAD, M., ALMELA, V, et al Auxin interaction with the vesicular-arbuscular mycorrhizal fungus Glomus intraradices Schenck \& Smith improves vegetative growth of two citrus rootstocks. Scientia Horticulturae, Amsterdan, v.66, p.77-83, 1996

EDRISS, M.H., DAVIS, R.M., BURGER, D.W. Increased growth responses of citrus by several species of mycorrhizal fungi. HortScience, Alexandria, v.19, n.4, p.537-539, 1984.

FISKE, C.H., SUBBAROW, Y. The colorimetric determination of phosphorus. Journal of Biological Chemistry, Bethesda, v.66, p.375-400, 1925.

HANLEY, K.M., GREENE, D.W. Gibberellin-like compunds from two ectomycorrhizal fungi and the $\mathrm{GA}_{3}$ response on Scoth pine seedlings. HortScience, Alexandria, v.22, p.591-594, 1987.

MOSSE, B. Mycorrhiza in a sustainable agriculture. Biological Agriculture and Horticulture, Oxon, v.3, p.191-209, 1986.

NEMEC, S. Glomus intraradix effects on citrus rootstocks seedling growth in various potting media. Journal of Agricultural Science, Cambridge, v.118, p.315-323, 1992.

PALAZZO, D., POMMERENING, B., VANADIA, S. Effects of soil sterilization and vesicular-arbuscular mycorriza on growth of sour orange (Citrus aurantium L.) seedlings. Proceedings of International Society of Citriculture, 7, Acireale, v.2, p.621623, 1992.

PHILLIPS, J.M., HAYMAN, D.S. Improved procedures for cleaning roots and staining parasitic and vesicular-arbuscular mycorrhizal fungi for rapid assessment of infection. Transactions of the British Mycological Society, Londres, v.55, p.158-161, 1970.

ROSE, S.L., YOUNGBERG, C.T. Tripartite associations in snowbrush (Ceanothis volutinus). Effect of vesicular-arbuscular mycorrhizae on growth, nodulation and nitrogen fixation. Canadian Journal of Botany, Ottawa, v.59, p.34-39, 1981.

SALISBURY, F.B., ROSS, C.W. Fisiología vegetal. México: Iberoamérica, 1994. 759p.

SOUZA, P.V.D., AGUSTÍ FONFRÍA, M., ABAD BERJÓN, M., et al. Interação entre auxinas de síntese e fungos micorrízicos arbusculares: influência sobre o desenvolvimento vegetativo de plântulas de laranjeira azeda (Citrus aurantium L.). Pesquisa Agropecuária Gaúcha, Porto Alegre, v.2, n.2, p.167-172, 1996.

SOUZA, P.V.D., ABAD BERJÓN, M., ALMELA ORENGA, V., et al. Desenvolvimento do citrange Troyer infectado com fungo micorrízico, em dois substratos de cultivo. Pesquisa Agropecuária Brasileira, Brasília, v.32, n.10, p.1039-1045, 1997.

TOBAR, R., AZCÓN, R., BAREA, J.M. Improved nitrogen uptake and transport from ${ }^{15} \mathrm{~N}$-labelled nitrate by external hyphae of arbuscular mycorrhiza under water-stressed condictions. New Phytologist, Cambridge, v.126, p.119-122, 1994. 